Journal of Computer Science 7 (6): 892-901, 2011

ISSN 1549-3636

(C) 2011 Science Publications

\title{
Energy Aware Multiple Constraints Quality of Service Routing Protocol with Dynamic Mobility Prediction for Mobile Ad hoc Networks
}

\author{
${ }^{1}$ Senthilkumar Maruthamuthu and ${ }^{2}$ Somasundaram Sankaralingam \\ ${ }^{1}$ Department of Computer Technology and Applications, \\ ${ }^{2}$ Department of Mathematics, \\ Coimbatore Institute of Technology, Coimbatore-641 014, Tamilnadu, India
}

\begin{abstract}
Problem statement: This study presents a source based reactive protocol called "Energy Aware Multiple Constraints QoS Routing Protocol with Dynamic Mobility Prediction (EMQRPDM)" for MANET. It is an enhanced version of our previous protocol called "Power aware multiple QoS constraints routing protocol with mobility prediction (PMQRPMP)". It addresses the issues such as routing, mobility and power management in MANET. Approach: EMQRPDM considers quality of service parameters namely delay, jitter, bandwidth and cost of each link on ' $n$ ' available paths and selects a stable optimal path between a source and a destination during path discovery. It checks a bandwidth constraint during route request. It also checks the energy level constraints for each node during route reply. EMQRPDM uses our new mobility prediction mechanism to determine the stability of link expiration time for each link of each path during route reply. It executes path maintenance procedure when the link between two nodes cuts off. It also considers a backup path during link failure. Results: EMQRPDM is compared with PMQRPMP. Even though the mobility speed of mobile nodes is increased to $10 \mathrm{~m} \mathrm{sec}^{-1}$, the success rate of data transmission in EMQRPDM is $74 \%$ which is still higher than PMQRPMP. During link failure using a backup path, the cost of control overhead in EMQRPDM is 0.272 which is lower than PMQRPMP when the number of mobile nodes is increased from 15-40. Conclusion/Recommendations: EMQRPDM selects an optimal path with good battery backup using energy level constraints. It selects a best stable optimal path using our new mobility prediction mechanism. EMQRPDM gives better Packet Delivery Ratio. It reduces the cost of control overhead using the bandwidth constraint and a backup path. It can be further enhanced as a reliable, secure, hybrid and multicast communication protocol for MANET.
\end{abstract}

Keywords: Energy level, QoS, routing protocol, mobility prediction, MANET, distributed ad hoc routing, bandwidth constraint, optimal path, Network Time Protocol (NTP), during route, mobile nodes

\section{INTRODUCTION}

Mobile Ad hoc Networks (MANETs) can be defined as the infrastructure less, self coordinated, suddenly created wireless networks with a set of mobile nodes. Some of the application areas of MANET are emergency search, military battlefields, rescue sites, meetings and classrooms where participants share information dynamically using their mobile nodes (AlHunaity et al., 2007). Some of main issues in MANET are routing, mobility management, reliability, power consumption and security. Quality of Service (QoS) can be defined as the service guarantee provided by the network to the user to meet a set of pre-specified service requirements while transporting a data packet from a source to destination.
This agreement is made based on the service metrics namely end-to-end delay, jitter, bandwidth and cost while transporting a data packet from a source node to destination node (Murad and Al-Mahadeen, 2007).

The QoS metrics can be classified as additive, concave, and multiplicative. Bandwidth is concave metric, while cost, delay, and jitter are additive metrics The QoS constraints can be formed based on the QoS metrics. They can be classified as time constraints, space constraints and frequency constraints (Senthilkumar et al., 2009). There can be ' $\mathrm{n}$ ' number of paths between a source and destination. The links on the paths are expected to satisfy the QoS constraints.

A path can be chosen as an optimal path, if it satisfies the QoS constraints. The essential task for Qos

Corresponding Author: Senthilkumar Maruthamuthu, Department of Computer Technology and Applications,

Coimbatore Institute of Technology, Coimbatore-641 014, Tamilnadu, India Tel: +91- 9443550935 
routing is to find a feasible path through the network between the source and destination that will have the necessary resources available to meet the QoS constraints (Murad and Al-Mahadeen, 2007).

The routing protocols in MANET can be categorized as proactive and reactive. In proactive routing, route discovery is easy but route maintenance is hard. In reactive routing, route discovery is hard but route maintenance is easy. Some of the QoS routing protocols for MANET are Core Extraction Distributed Ad hoc Routing (CEDAR), QoS-AODV (QAODV) and Ticket-Based Probing (TBP). CEDAR uses clustered network architecture and selects the core dynamically. In CEDAR (Sinha et al., 1999), there may be a risk that the core fails due to hardware and software problems. Since more data are routed through the core node, the core node suffers from heavy traffic. QAODV (Sinha et al., 1999) is a reactive routing protocol. In QAODV, route discovery consumes more time. Every intermediate node checks whether it can support for the specified QoS. TBP (Chen and Nahrstedt, 1999) is a multipath QoS routing scheme. In TBP, source sends N number of tickets to find $\mathrm{N}$ paths. There is no clear heuristic for computing tickets. Resource Reservation for one flow denies the availability of that resource for other flows.

Our previous protocol called PMQRPMP (Senthilkumar et al., 2009) adds a power constraint along with multiple QoS constraints mentioned in MQRPMP (Lian et al., 2007) to select a best routing path among multiple paths between a source and a destination as to increase Packet Delivery Ratio (PDR), reliability and efficiency of mobile communication. It collects the residual battery power of each node for each path; selects a path, which has nodes with good battery power for transmission to satisfy the power constraint. PMQRPMP uses the mobility prediction formula (Lian et al., 2007) to find the Link Expiration Time (LET) between two nodes. It has better PDR than MQRPMP and TBP. The cost of communication overhead in PMQRPMP is also less than TBP. During communication, there may be a chance for a mobile node to suddenly increase or decrease its speed or direction when it is moving. This can be known as dynamic mobility. PMQRPMP does not address the impact of dynamic mobility.

The proposed protocol EMQRPDM is the extension of our previous work PMQRPMP.

It is a source based reactive protocol for finding an optimal path which satisfies a set of link and node constraints. It has highest predicted LET value and good Energy Level (EL). EMQRPDM uses our new mobility prediction mechanism which finds LET and predicts the stability of LET based on dynamic mobility of nodes for finding an optimal path between two mobile nodes.

\section{MATERIALS AND METHODS}

Mobility prediction mechanism: The routing protocol in MANET uses the location information obtained from Global Positioning System (GPS) (Kaplan, 1996) to estimate LET of a link between two adjacent nodes. Based on this prediction, routes are reconfigured before they disconnect.

The routing protocol considers free space propagation model (Su et al., 2000). It is assumed that all nodes in the network have their clock synchronized [e.g., by using the Network Time Protocol (NTP) or the GPS clock itself]. Therefore, using the motion parameters such as speed, direction and communication distance of two neighbors, LET can be computed using the well-known mobility prediction formula. Assume that the two nodes $i$ and $j$ are within the transmission range $r$ of each other. Let (xi, yi) be the coordinate of mobile host $\mathrm{i}$ and (xj, yj) be that of mobile host $\mathrm{j}$. Also let vi and vj be the speeds and $\theta \mathrm{i}$ and $\theta \mathrm{j}$ be the moving directions of nodes $i$ and $j$, respectively.

LET $=\frac{-(a b+c a)+\sqrt{\left(\left(a^{2}+c^{2}\right) r^{2}-(a d-b c)\right)}}{\left(a^{2}+c^{2}\right)}$

Where:

$a=v i \cos \theta i-v j \cos \theta j ; b=x i-x j ;$

$\mathrm{c}=\mathrm{vi} \sin \theta \mathrm{i}-\mathrm{vj} \sin \theta \mathrm{j}$ and $\mathrm{d}=\mathrm{yi}-\mathrm{yj}$

Note that when $v i=v j$ and $\theta i=\theta j$, LET is set to $\infty$ without applying the above equation.

Impact of dynamic mobility of mobile nodes: The Eq. 1 can be used for identifying the stability of a link between two adjacent nodes. But if a node on a link suddenly alters its speed/direction or both, the LET associated with that link is also needed to be altered. This dynamic mobility feature of mobile nodes is not addressed in Eq. 1. It is analyzed as follows:

Let us assume that $\mathrm{i}$ and $\mathrm{j}$ are the two nodes of a link.

Case 1: Either $i$ or $j$ is expected to increase or decrease its speed, direction or both during mobility.

Case 2: Both mobile nodes $\mathrm{i}$ and $\mathrm{j}$ are expected to increase or decrease their speed/direction or both during mobility. 
In both the cases, due to high dynamism in mobility, the LET associated with that link is expected to be changeable. This in turn affects the stability of the link. This affects the stability of the entire path. Apart from this, even though the LET is high, if any one of the nodes or both the nodes of the corresponding link are not having sufficient EL, there may be a chance to lose at least a node in that link which in turn leads to non existence of the link. This affects the stability of the link. So the computed LET for that link is not optimum.

On the other hand, the nodes on a selected link may have good EL and they may forward many packets. If any one of the node or both the nodes on that link is cut off due to sudden alteration in its speed/direction or both during mobility, the PDR on that link is obviously getting reduced. This affects PDR on the selected path. Thus the LET in Eq. 1 is changeable during dynamic mobility.

Prediction of LET: The new mobility prediction formula for the proposed protocol for finding the stability of LET is given as follows. The duration of time required to lose the complete EL of a node is referred to as the lifetime of that node. A node loses its energy during its sleep mode, processing mode and transmission mode. So the accumulation of all the energy loss durations in these three modes determines the life time of a node. Let $\mathrm{X}$ be the time required to lose the energy at a constant rate during the sleep mode. Let $\mathrm{Y}$ be the time required to lose the energy to process ' $n$ ' packets of size ' $m$ '. Let $Z$ be the time required to lose the energy to transmit those ' $n$ ' packets of size ' $m$ '.

So the lifetime of a node ' $i$ ' can be computed using Eq. 2 as follows:

lifetime $(\mathrm{i})=\mathrm{X}+\mathrm{Y}+\mathrm{Z}$

Since the stability of the link $\mathrm{i}-\mathrm{j}$ is not only based on the calculated LET but also depends on the lifetime of nodes ' $\mathrm{i}$ ' and ' $\mathrm{j}$ ' associated with that link, the stability of the link (CurrentLET) is calculated using eq. 3 as follows:

CurrentLET $=$ Min(LET, lifetime(i), lifetime(j))

The CurrentLET in Eq. 3 can further be tuned based on the following two situations according to the above mentioned cases for dynamic mobility.

Firstly whenever the two nodes of a link become closer to each other the CurrentLET associated with that link also increases. Otherwise the two nodes of a link deviate from each other and the CurrentLET associated with that link also decreases. Secondly even though the lifetime of the nodes on the link is already considered for CurrentLET in Eq. 3, it is also necessary to check whether these two nodes on that link have enough EL for data transmission or not.

According to the first situation, in order to determine whether the nodes associated with a link moves closer to each other or deviates from each other, the calculated CurrentLET is compared with the recent past CurrentLET. If the CurrentLET $>=$ recent past LET then the mobile nodes are becoming closer to each other. This shows that the CurrentLET associated with that link is expected to be increasing in near future. So the CurrentLET can be further tuned by adding a positive value to it. Otherwise, if the CurrentLET < recent past LET then the mobile nodes are deviating from each other. This shows that the CurrentLET associated with that link is expected to be decreasing in near future. So the CurrentLET can be further tuned by adding a negative value to it.

According to the second situation, the EL of each node on a link is checked against the EL thresholds ELTH1 and EL-TH2 where EL-TH1 < EL-TH2. Based on these comparisons the nodes on a link can be classified as good, normal and weak. If a node has EL $\geq$ the ELTH2 then the node can handle heavy traffic for a longer duration. This kind of node can be assumed as a good node in communication. If a node has the EL-TH1 $\leq$ EL $<$ EL-TH2 then it is assumed as a normal node and can handle normal traffic. If a node has the EL $<$ EL-TH1 then it is assumed as a weak node. Based on the classification of nodes as good, normal and weak based on their EL, it is possible to tune the CurrentLET of the associated link. If any one of the node is a weak node on a link the CurrentLET of that link is not tuned for our protocol.

So according to the above two situations, the CurrentLET in Eq. 3 is tuned by a suitable variable called MAF (Mobility Adjustment Factor) during dynamic mobility. The LET computed by adding the value of MAF with CurrentLET is known as the PredictedLET. The PredictedLET value is computed using the Eq. 4 as follows.

PredictedLET $=$ CurrentLET + MAF

Since the CurrentLET is represented as the value of time, the MAF should also be represented as a value of time.

Simply assigning an arbitrary time value to MAF may not be realistic.

So the value of the tuning variable MAF can be computed using the equations from 5-8 as follows: 
Let T-TH1 (i) is the time duration required for a node 'i' can have EL up to EL-TH1. It can be calculated using Eq. 5 as follows:

$$
\mathrm{T}-\mathrm{TH1}(\mathrm{i})=\frac{\text { lifetime }(\mathrm{i})}{\mathrm{EL}} * \mathrm{EL}-\mathrm{TH} 1
$$

Let T-Above-TH1(i) be the time duration in which a node 'i' can have EL > EL-TH1. It can be calculated using Eq. 6 as follows:

$$
\mathrm{T}-\text { Above }-\mathrm{TH} 1(\mathrm{i})=\text { lifetime }(\mathrm{i})-\mathrm{T}-\mathrm{TH}(\mathrm{i})
$$

Let $\mathrm{T}$ (i) be the time duration in which a link $\mathrm{i}-\mathrm{j}$ is stable during CurrentLET based on the T-Above-TH1(i) such that $\mathrm{T}$ (i) $<=$ CurrentLET. The computation of $\mathrm{T}$ (i) is given in Eq. 7 as follows:

$$
\mathrm{T}(\mathrm{i})=\frac{\mathrm{T}-\text { Above }-\mathrm{TH1}(\mathrm{i})}{\text { Lifetime }(\mathrm{i})} * \text { CurrentLET }
$$

Likewise for the node ' $\mathrm{j}$ ', T-TH1(j), T-AboveTH1(j) and T(j) are also calculated. Let $T$ be the time duration in which a link $\mathrm{i}-\mathrm{j}$ is stable during CurrentLET based on the $\mathrm{T}(\mathrm{i})$ and $\mathrm{T}(\mathrm{j})$ such that $\mathrm{T}<=$ CurrentLET is given in Eq. 9 as follows:

$$
\mathrm{T}=\operatorname{Min}(\mathrm{T}(\mathrm{i}), \mathrm{T}(\mathrm{j}))
$$

The value of $T$ is used to determine the value of the tuning variable MAF. It is shown in Table 1. The Eq. 4 can be applied for different types of applications. The value of MAF for any link at a particular time can be either of the values in the set $=\{-5 \mathrm{~T},-4 \mathrm{~T},-3 \mathrm{~T},-2 \mathrm{~T},-1 \mathrm{~T}$, $1 \mathrm{~T}, 2 \mathrm{~T}, 3 \mathrm{~T}, 4 \mathrm{~T}, 5 \mathrm{~T}\}$. When both nodes are not altering their speed and direction, MAF value becomes 0 . If $\mathrm{MAF}=0$ then the PredictedLET is made equal to the CurrentLET of that link.

For example, let us consider the lifetime(i) $=14$ Secs, lifetime $(\mathrm{j})=15$ Secs, CurrentLET $(\mathrm{i}-\mathrm{j})=3$ Secs, $\mathrm{EL}(\mathrm{i})=90 \%, \mathrm{EL}(\mathrm{j})=80 \%$ and the ELD-TH $1=60 \%$.

Therefore:

T-TH1 (i) $=(14 / 90) * 60=9.333$ Secs

T-TH1 $(\mathrm{j})=(15 / 80)^{*} 60=11.25$ Secs

T-Above-TH1 (i) $=14-\mathrm{T}-\mathrm{TH} 1(\mathrm{i})=4.666 \mathrm{Secs}$

T-Above-TH1 (j) $=15$-T-TH1 $(\mathrm{j})=3.75$ Secs

$\mathrm{T}$ (i) $=(4.666 / 14) * 3=0.9998$ Secs

$\mathrm{T}(\mathrm{j})=(3.75 / 15) * 3=0.75$ Secs

$\mathrm{T}=\operatorname{minimum}(0.9998,0.75)=0.75$
Proposed network model: The proposed network model in MANET can be denoted by $\mathrm{G}=\{\mathrm{V}, \mathrm{E}\}$ where $\mathrm{V}$ is the set of interconnected nodes and $\mathrm{E}$ is the set of full-duplex directed wireless communication links. The network model considers the existence of multiple paths between any two nodes where each link on each path considers the QoS metrics namely Delay (D), Jitter (J), Bandwidth (B), Cost(C) and HopCount(HC). The Cost function (C) can be modeled as a linear function as shown below where $b$ is the fixed cost of channel acquisition and $\mathrm{m}$ is the incremental cost proportional to the size of network layer packet to be transmitted between the nodes $i$ and $j$. It can be the transmission cost of the packet. This model also considers the EL of each node $\left(\mathrm{V}_{\mathrm{i}}\right)$ on each path, which meets the power thresholds EL-TH1 and EL-TH2 where EL-TH1< EL$\mathrm{TH} 2$. The model expects that the EL of each node and its corresponding path should satisfy at least the minimum threshold EL-TH1. The EL of each node is the residual battery backup, which is collected and summed up for each routing path. The EL of each path is the Avg $\left(\sum \mathrm{EL}\left(\mathrm{V}_{\mathrm{i}}\right)\right)$. This model also includes the parameter called PredictedLET for the selection of an optimal path. Among the existence of multiple paths $\left(\mathrm{P}_{1}, \mathrm{P}_{2}, \mathrm{P}_{3} \ldots\right.$ and $\left.\mathrm{P}_{\mathrm{n}}\right)$ for a source to destination, an optimal path $P_{k}$ is selected which satisfies all the above said constraints. So the problem of multiple QoS constraints with power awareness and new mobility prediction mechanism for the selection of an optimal path can be defined as follows:

Select $\mathrm{P}_{\mathrm{k}}$ among $\left(\mathrm{P}_{1}, \mathrm{P}_{2}, \mathrm{P}_{3} \ldots\right.$ and $\left.\mathrm{P}_{\mathrm{n}}\right)$

where, PredictedLET $>0$ Such that:

$$
\begin{aligned}
& \sum \mathrm{Dij} \leq \mathrm{Dc} \\
& \sum \mathrm{Jij} \leq \mathrm{Jc} \\
& \mathrm{Bij} \geq \mathrm{Bc} \\
& \mathrm{Cij}=\mathrm{m} * \text { size }+\mathrm{b} \\
& \sum \mathrm{Cij} \leq \mathrm{Cc} \\
& \mathrm{HCsum} \leq \mathrm{Hc} \\
& \text { EL (Vi) } \geq \text { EL-TH1 and Avg }\left(\sum E L(V i)\right) \geq \text { EL-TH1 } \\
& \text { PredictedLET }\left(\mathrm{P}_{\mathrm{k}}\right) \geq \operatorname{PredictedLET}\left(\mathrm{P}_{1}, \mathrm{P}_{2}, \mathrm{P}_{3} \ldots \text { and } \mathrm{P}_{\mathrm{n}}\right)
\end{aligned}
$$

Energy aware QoS Routing Protocol with Dynamic Mobility Prediction (EMQRPDM): The path discovery and path maintenance procedures for the proposed protocol are given as follows.

During the path discovery, an optimal path is selected based on multiple QoS constraints and power constraint using our new mobility prediction formula. The path with highest battery power and highest PredictedLET is considered as stable optimal path. 
Table 1: MAF computation for each link

\begin{tabular}{|c|c|c|c|c|c|c|}
\hline $\begin{array}{l}\text { Type of the } \\
\text { source }\end{array}$ & Type of the destination & Moving node(s) & $\begin{array}{l}\text { Closer to } \\
\text { other node }\end{array}$ & $\begin{array}{l}\text { Deviates from } \\
\text { other node }\end{array}$ & $\begin{array}{l}\text { Gain(G) or } \\
\text { Loss (L) }\end{array}$ & MAF \\
\hline Normal node & Normal node or Good node & Source/ Destination & Yes & No & $\mathrm{G}$ & $\mathrm{T}$ \\
\hline Good node & Normal node or Good node & Source/ Destination & Yes & No & GG & $2 \mathrm{~T}$ \\
\hline Normal node & Normal node & Source and Destination & Yes & No & GGG & $3 \mathrm{~T}$ \\
\hline Normal node & Good node & Source and Destination & Yes & No & GGGG & $4 \mathrm{~T}$ \\
\hline Good node & Good node & Source and Destination & Yes & No & GGGGG & $5 \mathrm{~T}$ \\
\hline Normal node & Normal node or Good node & Source/ Destination & No & Yes & $\mathrm{L}$ & $-T$ \\
\hline Good node & Normal node or Good node & Source/ Destination & No & Yes & LL & $-2 \mathrm{~T}$ \\
\hline Normal node & Node & Source/ Destination & No & Yes & LLL & $-3 \mathrm{~T}$ \\
\hline Normal node & Good node & Source and Destination & No & Yes & LLLL & $-4 \mathrm{~T}$ \\
\hline Good node & Good node & Source and Destination & No & Yes & LLLLL & $-5 \mathrm{~T}$ \\
\hline
\end{tabular}

Path discovery: In this protocol, the source broadcasts a Route Request packet (RREQ) with the fields Sourceaddress, Destination-address, Packet type, RouteRequestId, Bc, EL-TH1 and EL-TH2. If an intermediate node (1) receives the RREQ then it forwards the received RREQ on each outgoing link only when bandwidth of the link is $>\mathrm{Bc}$. This reduces the number of RREQs during route discovery. This in turn reduces control overhead.

If the destination node receives a duplicate RREQ then received RREQ is discarded. Otherwise, the destination node constructs a Route Reply packet (RREP). The fields in a RREP are as follows: Sourceaddress, Destination-address, Packet type, RouteReplyId, EL-TH1, EL-TH2, D, J, B, C, CurrentLET, PredictedLET, EL, HC, Speed and Direction. The destination node sets 0 to the fields D, J, C, CurrentLET, PredictedLET and HC in that RREP. It copies EL-TH1 and EL-TH2 from received RREQ and includes its moving direction and speed into the RREP. Then the destination node sends RREP towards the source. Our protocol maintains a table called LETtable at each intermediate node with the fields namely Source of the link, Destination of the link, CurrentLET and PredictedLET. Initially, LETtable is empty. Whenever an intermediate node ' $i$ ' receives a route reply from a node ' $\mathrm{j}$ ', it checks whether EL(i) > = EL-TH1. If it so, it computes CurrentLET for the link i-j using the eq. 4 and checks whether the corresponding entry is found in the LETtable for that link.

If the entry is not found, then the fields CurrentLET and PredictedLET in LETtable are set to the currently computed CurrentLET and zero respectively. If the entry is found, then the CurrentLET is compared with the existing CurrentLET (recent past CurrentLET) in LETtable to check whether ' $i$ ' and ' $j$ ' are becoming closer to each other or not. It also compares ELs of ' $\mathrm{i}$ ' and ' $\mathrm{j}$ ' against the EL-TH2 and classifies whether they are good or normal. Based on the closeness and the classifications, the value of MAF is determined to tune the value of CurrentLET to calculate the PredictedLET.
Then the fields CurrentLET and PredictedLET in LETtable are set to the calculated CurrentLET and PredictedLET respectively. Then intermediate node ' $i$ ' updates the fields D, J, B, C, CurrentLET, EL, PredictedLET, HC, Speed and Direction in the new RREP along with EL-TH1 and EL-TH2 copied from received RREP. Then the new RREP is forwarded towards the source.

The source maintains a table called MetricsTable with the fields namely Dsum, Jsum, Csum, LET, ELsum, HCsum and PredictedLET. It updates all these fields using D, J, C, CurrentLET, PredictedLET, EL and $\mathrm{HC}$ received from each RREP. Then the source sorts the MetricsTable based on PredictedLET. It compares Dsum, Jsum, Csum, HCsum of each RREP against the thresholds Dc, Jc, Cc and Hc. If the comparison is successful then the route mentioned by that corresponding RREP is included in a table called RouteSelectionTable at the source. Likewise the source gathers all satisfied RREPs into RouteSelectionTable and identifies an optimal path among ' $n$ ' available paths which meet the mentioned QoS constraints to destination. The route discovery procedure for the proposed protocol is given as follows:

\section{Procedure for Source (S):}

If Source $S$ has no Paths to Destination D

Set the QoS Constraints

Construct and Broadcast RREQ

Execute Route Reply Handling Procedure

Execute Route maintenance

End if

\section{Route request handling procedure:}

If it is an intermediate node I

If the received RREQ is not duplicate

If $\left(\mathrm{B}_{\mathrm{ij}}>=\mathrm{B}_{\mathrm{c}}\right)$

Forward RREQ

End if

End if

End if 
If it is destination D

If the received RREQ is not duplicate

Execute Route Reply Handling Procedure

End if

\section{End if}

\section{Route reply handling procedure:}

If it is destination $\mathrm{D}$

If the received RREQ is not duplicate

Set D, J, B, C, CurrentLET, PredictedLET and HC to 0

Get its speed and direction from GPS

Construct RREP including the fields

EL-TH1, EL-TH2

Forward RREP towards $\mathrm{S}$

End if End if

If it is an intermediate node I

If the received RREP is not duplicate and EL $\left(\mathrm{V}_{\mathrm{i}}\right)$ $>=$ EL-TH1

$\mathrm{D}=$ ReceivedD + CurrentD

$\mathrm{J}=$ Received $\mathbf{J}+$ Current $\mathbf{J}$

$\mathrm{C}=$ ReceivedC + CurrentC

$\mathrm{EL}=$ ReceivedEL + CurrentEL

$\mathrm{HC}=$ ReceivedHC +1

CurrentLET $=$ Min(ReceivedCurrentLET, CurrentLET)

Get its speed and Direction from GPS

Calculate predicted LET

Construct RREP including the fields

EL-TH1, EL-TH2 and Forward RREP to S End if

End if

If the node is $\mathrm{S}$

Receive the RREPs

Collect the paths to D

If the collection is not NULL Sort all the paths based on their predicted LET

For each Path $\mathrm{P}_{\mathrm{i}}$

$$
\begin{aligned}
& \text { If } \sum \mathrm{D}_{\mathrm{ij}}<=\mathrm{D}_{\mathrm{c}}, \sum \mathrm{J}_{\mathrm{ij}}<=\mathrm{J}_{\mathrm{c}}, \sum \mathrm{C}_{\mathrm{ij}}<=\mathrm{C}_{\mathrm{c}}, \\
& \mathrm{HC}_{\text {sum }}<=\mathrm{H}_{\mathrm{c}} \text { and PredictedLET }>0 \\
& \text { Select the Path } \mathrm{P}_{\mathrm{i}} \\
& \text { Put the path in Route selectiontable } \\
& \text { Else Delete routing path } \\
& \text { Endif }
\end{aligned}
$$

End for

For each Path $\mathrm{P}_{\mathrm{i}}$ in route selectiontable and $\mathrm{P}_{\mathrm{n}}$ )

If predicted $\operatorname{LET}\left(\mathrm{P}_{\mathrm{i}}\right) \geq$ predicted $\operatorname{LET}\left(\mathrm{P}_{1}, \mathrm{P}_{2}, \mathrm{P}_{3} \ldots\right.$

Select $\mathrm{P}_{\mathrm{i}}$ for transmission

Endif

End for

End if

End if
Path maintenance: Due to the dynamic changes of network topology and limitation of network resources, the computed optimal route often gets invalidated. When the link is cut off, the upstream node sends Route Reconstruction packet (RREC) to the source. Then the source once again starts the route discovery procedure. If the source receives RREP and RREC at the same time, it deals with the RREC.

\section{Route maintenance procedure by the intermediate node:}

If the link is cut off with its neighbor

Construct and Send RREC to $\mathrm{S}$

End if

If the RREC is received from its neighbor

Forward the RREC to $\mathrm{S}$

End if

\section{Route maintenance procedure by the source node:}

If the RREC is received from any I

If backup path is available

$\begin{array}{ll} & \text { Route the packets via backup path } \\ \text { Else } & \text { Broadcast RREQ } \\ \text { End if } & \end{array}$

End if

If the RREC and RREP is received at the same time from any $\mathrm{I}$

End if

Broadcast New RREQ

Illustration: Figure 1 depicts a graph with QoS metrics for links in EMQRPDM. Let Dc $=15, \mathrm{Jc}=30$, $\mathrm{Bc}=35, \mathrm{Cc}=40$ and $\mathrm{Hc}=5$. Let the EL-TH1 and ELTH2 are 60 and 90. Let the energy levels of the nodes 1 , 2, 3, 4, 5 and 6 are 90, 85, 95, 95, 98 and 85 respectively. Let the route from the node 1 to destination 6 is requested. According to multiple QoS constraints, EL constraints and PredictedLET the routes are identified.

In this example, the path $\mathrm{P}_{1}(1,2,4,6)$ does not satisfy delay constraint. The paths $\mathrm{P}_{2}(1,3,5,4,6), \mathrm{P}_{3}$ $(1,3,2,4,5,6), \mathrm{P}_{4}(1,2,4,5,6)$ and $\mathrm{P}_{5}(1,2,3,5,4,6)$ do not satisfy delay constraint, bandwidth and cost constraints respectively. But the paths $\mathrm{P}_{6}(1,3,5,6)$ and $\mathrm{P}_{7}$ $(1,3,2,4,6)$ satisfy delay, jitter, bandwidth, cost and hop constraints. The paths are at least expected to meet the basic EL constraint EL(Vi) $>=$ EL-TH1. They may meet or may not meet the other EL constraint EL(Vi) > $=$ EL-TH2. According to Fig. 1 all the above-mentioned paths satisfy this basic EL constraint. 
Table 2 shows the details of recent past CurrentLET values for the nodes in the path $\mathrm{P}_{6}$ and $\mathrm{P}_{7}$ based on the Eq. 3 along with their EL values before mobility of nodes. Since CurrentLET is a concave metric, the CurrentLET of the paths $\mathrm{P}_{6}$ and $\mathrm{P}_{7}$ are computed as 1.3 and 1.271 respectively and these CurrentLETs are $>0$.

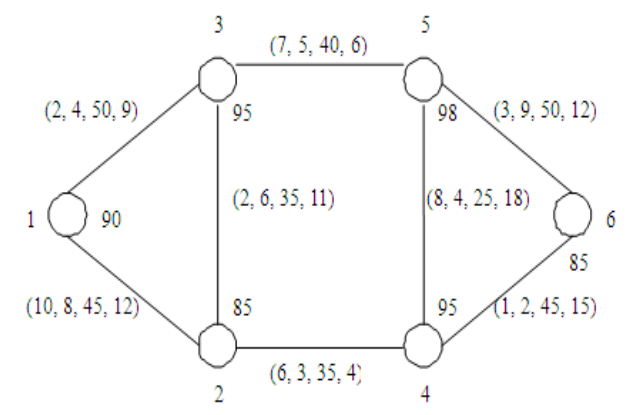

Fig. 1: Example of multiple QoS constraints network

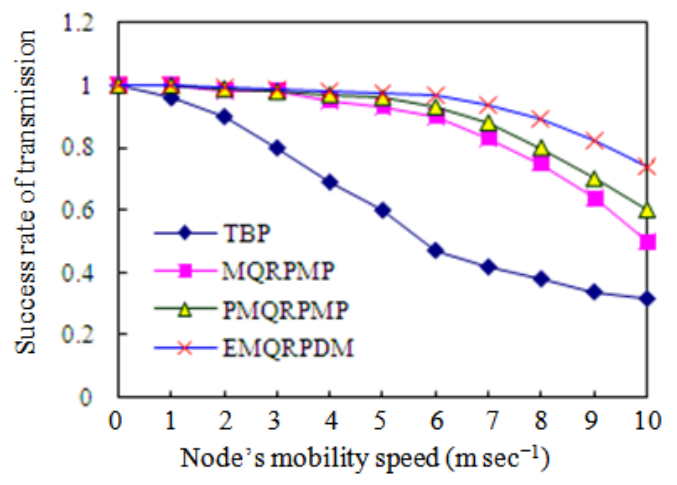

Fig. 2: Success rate of data transmission and node's mobility speed

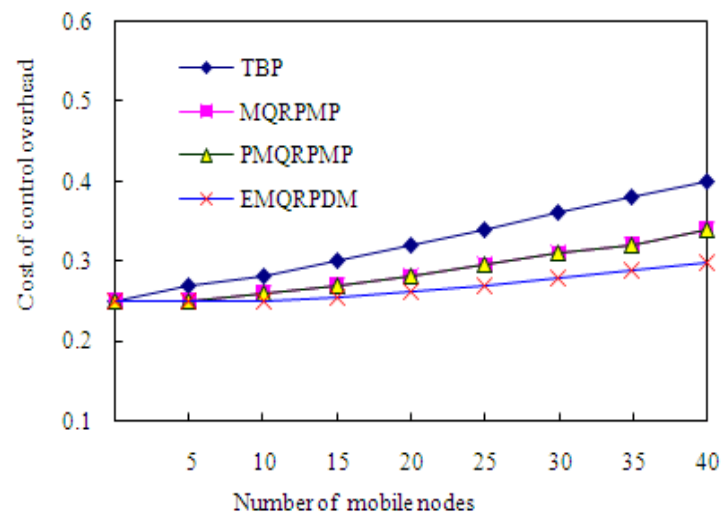

Fig. 3: Cost of control overhead and number of mobile nodes
Table 3 shows the Current LET for the same paths $\mathrm{P}_{6}$ and $\mathrm{P}_{7}$ based on the equation 3 after mobility of nodes along with their respective changes in EL. It should be noted that the lifetime of each node for each link are $>$ Current LET for the computation of Table 2 and Table 3. Table 4 shows the calculation of time duration $\mathrm{T}$ in which each link on $\mathrm{P}_{6}$ and $\mathrm{P}_{7}$ are stable during CurrentLET to determine MAF of those paths based on the equations from 5-8. Table 5 shows the PredictedLET values for each link of $\mathrm{P}_{6}$ and $\mathrm{P}_{7}$ after tuning the CurrentLET (shown in Table 3) based on the calculated MAF values using equation 4 after mobility of nodes. The Predicted LET of the paths $\mathrm{P}_{6}$ and $\mathrm{P}_{7}$ are 2.407 and 2.311 and are $>0$. So the paths $P_{6}$ and $P_{7}$ are considered for route selection.

Table 6 is the Metrics Table used at the source. It contains the values of Dsum, Jsum, Csum, LET, EL sum, HC sum and Predicted LET received from the two route replies for the paths $\mathrm{P}_{6}(1,3,5,6)$ and $\mathrm{P}_{7}(1,3,2,4,6)$ respectively. This Metrics Table is sorted based on the Predicted LET. From the Table 2-5 it is clearly understood that the Predicted LETs of $\mathrm{P}_{6}$ and $\mathrm{P}_{7}$ are higher than their respective recent past Current LETs of Table 2 after mobility. This shows that these two paths $\mathrm{P}_{6}$ and $\mathrm{P}_{7}$ will be more stable and existing for longer duration till the link is cut off. Therefore the paths $\mathrm{P}_{6}$ and $\mathrm{P}_{7}$ are selected as the optimal paths for data transmission and included in the Route Selection Table as shown in Table 7. Since the Predicted LET of $\mathrm{P}_{6}$ in Route Selection Table is $>$ the Predicted LET of $\mathrm{P}_{7}$, the path $\mathrm{P}_{6}$ is selected as the most optimal path for data transmission

Simulation setup: The protocol is simulated in ns2 (Fall, 2001). The simulation parameters and their values are shown in the Table 8.

\section{RESULTS}

The metrics used for evaluating all these protocols are success rate of data transmission, cost of control overhead. These metrics were compared with the other metrics namely mobility speed and the number of mobile nodes. Our protocol is compared with PMQRPMP, MQRPMP and TBP. This is shown in Fig. 2-4. The Fig. 2 shows the comparison of success rate of data transmission along with the node's mobility speed. Fig. 3 shows the comparison of number of nodes with cost of control overhead incurred during transmission for EMQRPDM, MQRPMP and TBP. In Fig. 4, the comparison of number of nodes with cost of control overhead incurred is shown for EMQRPDM, PMQRPMP, MQRPMP and TBP during link failure while considering backup path. 
J. Computer Sci., 7 (6): 892-901, 2011

Table 2: Recent past current LET for $\mathrm{P}_{6}$ and $\mathrm{P}_{7}$ before mobility based on Eq. 3

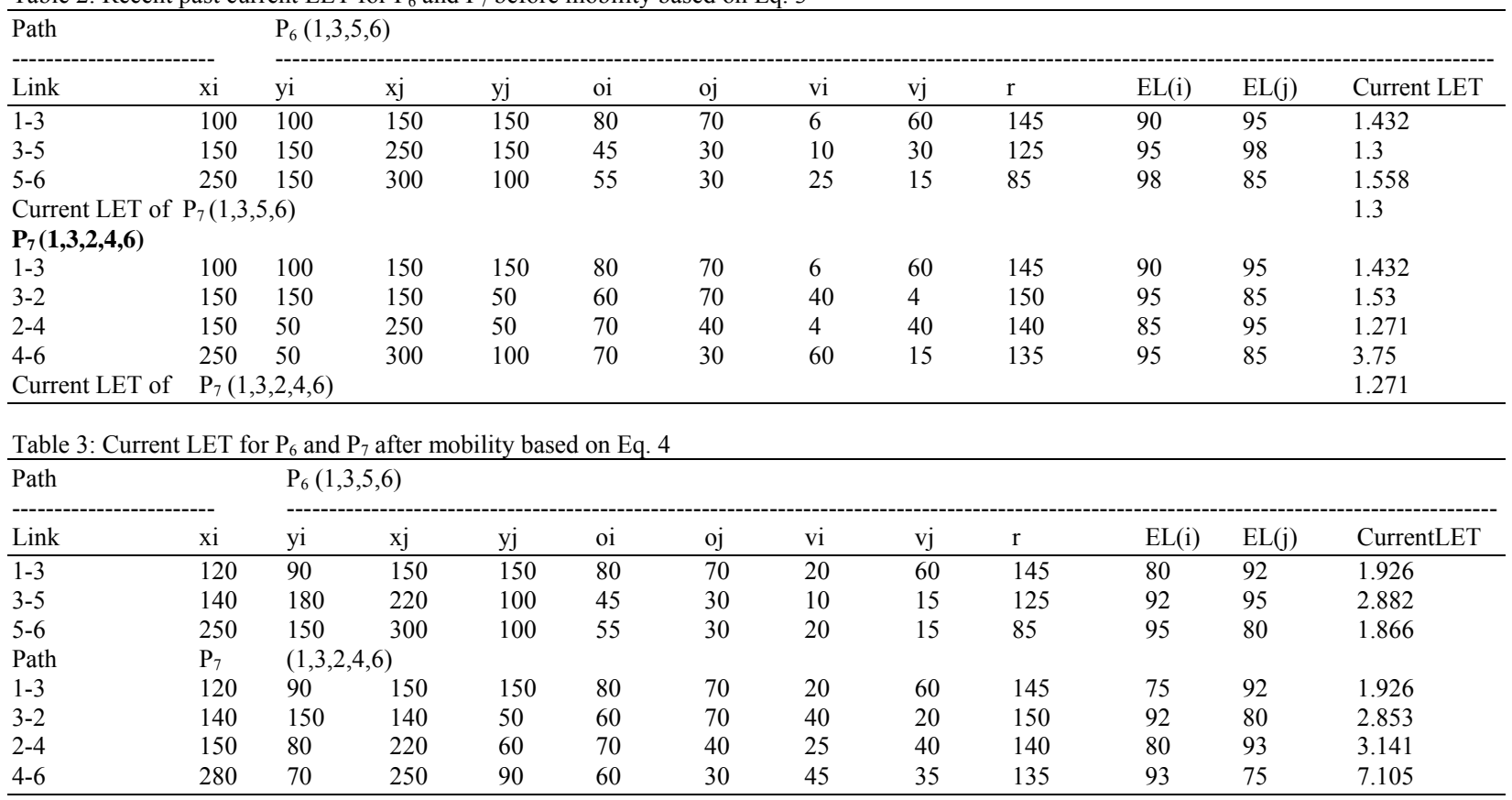

Table 4: Calculation of $\mathrm{T}$ to find MAF for paths $\mathrm{P}_{6}$ and $\mathrm{P}_{7}$ $\mathrm{P}_{6}(1,3,5,6)$ Based on EL-TH1 $=60$ and EL-TH2 $=90$

\begin{tabular}{|c|c|c|c|c|c|c|c|c|c|}
\hline $\begin{array}{l}\text { Path } \\
\text { Link }\end{array}$ & Node & EL & Speed change & $\begin{array}{l}\text { Current LET } \geq \text { Recent } \\
\text { past current LET }\end{array}$ & Life time & T-TH1 & T-ABOVE-TH1 & T(Node) & $\mathrm{T}$ \\
\hline \multirow[t]{2}{*}{$1-3$} & 1 & 80 & $\mathrm{Y}$ & $1.926>1.432$ & 14 & 10.5 & 3.5 & 0.481 & 0.481 \\
\hline & 3 & 92 & $\mathrm{~N}$ & & 15 & 9.782 & 5.217 & 0.669 & \\
\hline \multirow[t]{2}{*}{$3-5$} & 3 & 92 & $\mathrm{~N}$ & $2.882>1.3$ & 20 & 13.043 & 6.957 & 1.002 & 1.002 \\
\hline & 5 & 95 & $\mathrm{Y}$ & & 17 & 10.736 & 6.264 & 1.061 & \\
\hline \multirow[t]{2}{*}{$5-6$} & 5 & 95 & $\mathrm{Y}$ & $1.866>1.558$ & 16 & 10.105 & 5.895 & 0.687 & 0.466 \\
\hline & 6 & 80 & $\mathrm{~N}$ & & 18 & 13.5 & 4.5 & 0.466 & \\
\hline \multicolumn{10}{|l|}{$\mathbf{P}_{7}$} \\
\hline \multirow[t]{2}{*}{$1-3$} & 1 & 75 & $\mathrm{Y}$ & $1.926>1.432$ & 12 & $9002 \mathrm{E} 6$ & 2.4 & 0.385 & 0.385 \\
\hline & 3 & 92 & $\mathrm{~N}$ & & 15 & 9.782 & 5.218 & 0.669 & \\
\hline \multirow[t]{2}{*}{$3-2$} & 3 & 92 & $\mathrm{~N}$ & $2.853>1.53$ & 20 & 13.043 & 6.957 & 0.992 & 0.713 \\
\hline & 2 & 80 & $\mathrm{Y}$ & & 19 & 14.25 & 4.75 & 0.713 & \\
\hline \multirow[t]{2}{*}{$2-4$} & 2 & 80 & $\mathrm{Y}$ & $3.141>1.271$ & 19 & 14.25 & 4.75 & 0.785 & 0.785 \\
\hline & 4 & 93 & $\mathrm{~N}$ & & 17 & 10.967 & 6.033 & 1.114 & \\
\hline \multirow[t]{2}{*}{$4-6$} & 4 & 93 & $\mathrm{Y}$ & $7.105>3.75$ & 17 & 10.967 & 6.033 & 2.521 & 1.421 \\
\hline & 6 & 75 & $\mathrm{Y}$ & & 11 & 8.8 & 2.2 & 1.421 & \\
\hline
\end{tabular}

Table 5: Predicted LET for paths $\mathrm{P}_{6}$ and $\mathrm{P}_{7}$ based on Eq. 4

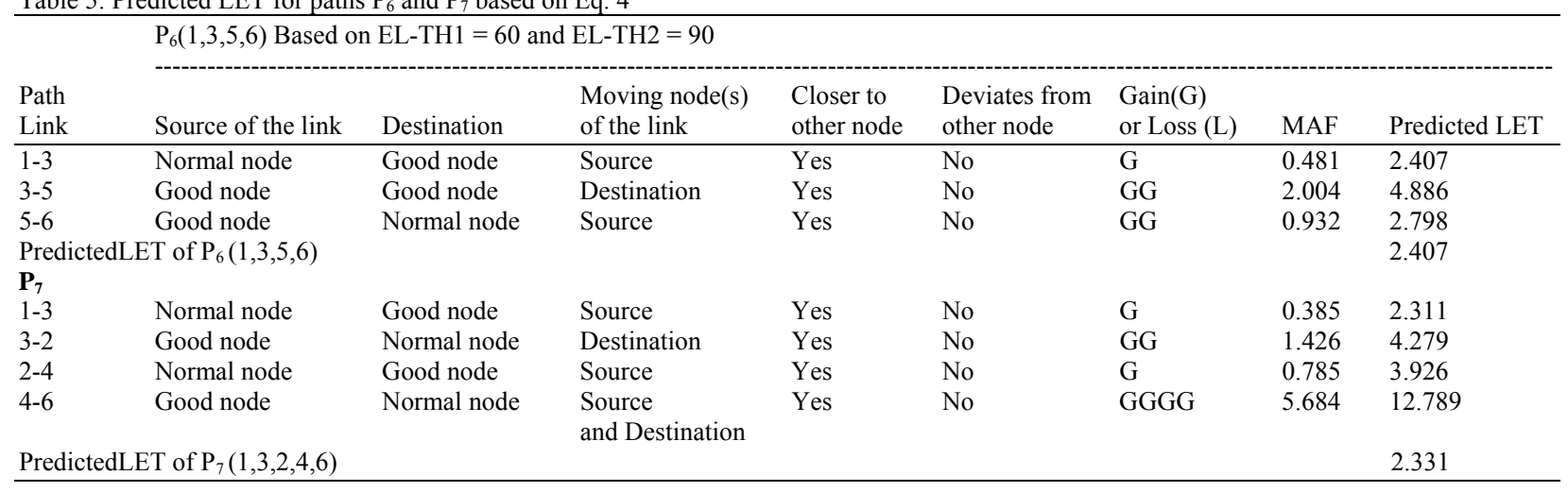


Table 6: Metrics table at source

\begin{tabular}{llllllll}
\hline & & & & & \multicolumn{3}{c}{ Predicted } \\
Path & $\mathrm{D}_{\text {sum }}$ & $\mathrm{J}_{\text {sum }}$ & $\mathrm{C}_{\text {sum }}$ & LET & $\mathrm{EL}_{\text {sum }}$ & LET & $\mathrm{HC}_{\text {sum }}$ \\
\hline $\mathrm{P}_{6}(1,3,5,6)$ & 12 & 18 & 27 & 1.3 & 267 & 2.407 & 3 \\
$\mathrm{P}_{7}(1,3,2,4,6)$ & 11 & 15 & 39 & 1.271 & 340 & 2.311 & 4 \\
\hline
\end{tabular}

Table 7: Route selectiont able

\begin{tabular}{lll}
\hline Path & Avg $\left(\sum \mathrm{EL}(\mathrm{Vi})\right)($ ie., EL(Pi)) & Optimal path \\
\hline $\mathrm{P}_{6}(1,3,5,6)$ & 89 & Yes \\
$\mathrm{P}_{7}(1,3,2,4,6)$ & 85 & Yes \\
\hline
\end{tabular}

Table 8: Simulation scenario

\begin{tabular}{ll}
\hline Simulation parameters & Given values \\
\hline MAC Layer (DCF) & IEEE 802.11 \\
Simulation area & $1 \mathrm{~km} * 1 \mathrm{~km}$ \\
Simulation time & $500 \mathrm{~s}$ \\
Number of mobile nodes & 45 \\
Node mobility speed & $0-10 \mathrm{~m} \mathrm{~s}$ \\
Node moving Pattern & Random way point \\
Traffic type & CBR \\
Packet size & 512 bytes \\
Transmission range & $250 \mathrm{~m}$ \\
\hline
\end{tabular}

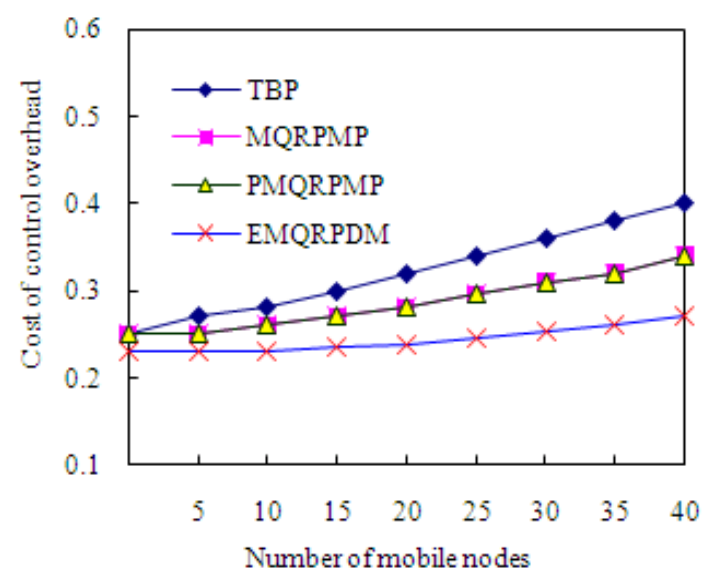

Fig. 4: Cost of control overhead and number of mobile nodes (during backup).

\section{DISCUSSION}

The Fig. 2 shows the comparison of success rate of data transmission along with the node's mobility speed. When the node's mobility speed is $3 \mathrm{~m} / \mathrm{s}$, the success rate of data transmission of MQRPMP and PMQRPMP reaches the value 0.98 which is higher than the TBP value 0.8 , but lower than the EMQRPDM value 0.987 . While increasing the node's mobility speed above 3 $\mathrm{m} / \mathrm{s}$, the performance of PMQRPMP, MQRPMP and TBP is drastically going down. But among them, in EMQRPDM the success rate of data transmission is increasing and it still higher than the others. It reaches 0.74 if node's mobility speed is 10 , due to PredictedLET computation.
Figure 3, shows the comparison of number of nodes with cost of control overhead incurred during transmission for EMQRPDM, MQRPMP and TBP. When increasing the number of nodes in communication, the cost of transmitting control packets also increases. Since PMQRPMP collects delay, jitter, bandwidth, cost and the energy level of each node along the path during route reply as exactly in MQRPMP, there is no performance difference between PMQRPMP and MQRPMP.

But in EMQRPDM due to the use of new mobility prediction formula and energy level constraints, the cost of control overhead is 0.255 which is lesser than the values $0.27,0.27$ and 0.3 for PMQRPMP, MQRPMP and TBP respectively when the number of mobile nodes is 15. As well as the cost of control overhead is still 0.297 which is very lesser than the values $0.34,0.34$ and 0.4 for PMQRPMP, MQRPMP and TBP respectively when the number of mobile nodes is increased to 40.This is shown in Fig. 3.

In Fig. 4, the comparison of number of nodes with cost of control overhead incurred during transmission for EMQRPDM, PMQRPMP, MQRPMP and TBP is shown. During link failure and backup path, the cost of transmitting control packets is reduced drastically in EMQRPDM than PMQRPMP, MQRPMP and TBP. It is 0.23 when the numbers of mobile nodes are 5, 10 and 15 in EMQRPDM which is lower than PMQRPMP, MQRPMP and TBP. It is becoming just 0.272 in EMQRPDM, if number of mobile nodes are 40 which is very lower than the values $0.34,0.34$ and 0.4 for PMQRPMP, MQRPMP and TBP respectively when the number of mobile nodes is increased to 40 due to the new mobility prediction formula and consideration of backup path.

\section{CONCLUSION}

This study discusses the new protocol EMQRPDM with multiple QoS constraints between source and destination. The main advantage of this protocol is that it considers power constraint for nodes for efficient packet transmission. It uses our new mobility prediction formula for PredictedLET calculation to select a stable path with minimal cost. The EMQRPDM provides a quick response to changes in the network reduces the waste of network resources and produces significant improvement in data transmission rate and hence reduces control overhead for reconstructing a routing path.

Future work in this direction can be the enhancement of EMQRPDM as a reliable and secure routing protocol by adding new constraints. There is the 
possibility of identifying more number of optimal stable paths in EMQRPDM.

So, this protocol can be enhanced a multipath routing protocol along with an intelligent load distribution algorithm. Based on the residual battery backup of mobile node on the selected route, the behavior of a mobile node can be changed from reactive to proactive and vice versa. So this protocol can also be enhanced as a hybrid routing protocol which in turn increases PDR considerably. Since MANET applications lend themselves well to multicast operations, this protocol can also be further extended as a multicast communication protocol.

\section{REFERENCES}

Al-Hunaity, M.F., S.A. Najim and I.M. El-Emary, 2007. A comparative study between various protocols of MANET networks. Am. J. Applied Sci., $\quad 4:$ 663-665. DOI: 10.3844/ajassp.2007.663.665

Chen, S. and K. Nahrstedt, 1999. Distributed quality-ofservice in ad hoc networks. IEEE J. Selec. Areas Commun., 17: 1488-1505. DOI: $10.1109 / 49.780354$

Fall, K., 2001. The NS Manual, the VINT Project, a Collaboration between Researchers at UC Berkeley, LBL, USC/ISI and Xerox PARC. http://www.isi.edu/nsnam/ns

Kaplan, E.D., 1996. Understanding the GPS: Principles and Applications. Artech House, Boston. http://www.navtechgps.com/Downloads/1024.PDF
Lian, J., L. Li and X. Zhu, 2007. A multiple QOS constraints routing protocol based on mobile predicting in ad hoc network. Proceedings of IEEE International Conference on Wireless Communications, Networking and Mobile Computing, Sep. 21-25, Shanghai, pp: 1608-1611. DOI: 10.1109/WICOM.2007.405

Murad, A.M. and B. Al-Mahadeen, 2007. Adding quality of service extensions to the enhanced associativity based routing protocol for Mobile Ad Hoc Networks (MANET). Am. J. Applied Sci., 4: 876-881. DOI: 10.3844/ajassp.2007.874.879

Senthilkumar, M., S. Somasundaram and R. Amuthakkannan, 2009. Power aware multiple QoS constraints routing protocol with mobility prediction for MANET. Int. J. Inform. Syst. Change Manage., 4: 156-170. DOI: 10.1504/IJISCM.2009.032754.

Sinha, P., R. Sivakumar and V. Bharghavan, 1999. CEDAR: A core extraction distributed ad hoc routing algorithm. IEEE J. Selec. Areas Commun., Vol. 17, Issue 8, pp. 1454-1465. DOI: $10.1109 / 49.779926$

Su, W., S.J. Lee and M. Gerla, 2000. Mobility prediction in wireless networks. Proceedings of 21st Century Military Communications Conference, Oct. 22-25, IEEE Xplore Press, Los Angeles, CA, USA, Vol 1, pp: 491-495. DOI: 10.1109/MILCOM.2000.905001 